\title{
Blood Pressure Changes during Pregnancy: Impact of Race, Body Mass Index, and Weight Gain
}

\author{
Urania Magriples, M.D. ${ }^{1}$, Marcella H. Boynton, Ph.D. ${ }^{2}$, Trace S. Kershaw, Ph.D. ${ }^{3}$, Sharon \\ Schindler Rising, C.N.M., M.S.N. ${ }^{4}$, and Jeannette R. Ickovics, Ph.D. ${ }^{3}$
}

${ }^{1}$ Department of Obstetrics and Gynecology, Yale University School of Medicine, New Haven, Connecticut ${ }^{2}$ Center for Child and Family Policy, Duke University, Durham, North Carolina ${ }^{3}$ Yale School of Public Health, New Haven, Connecticut ${ }^{4}$ Centering Healthcare Institute, Silver Spring, Maryland

\section{Abstract \\ Objective-To investigate the effect of race, body mass index (BMI), and weight gain on blood pressure in pregnancy and postpartum.}

Study Design-Secondary analysis of pregnant women aged 14 to 25 who received prenatal care at a university-affiliated public clinic in New Haven, Connecticut and delivered singleton term infants $(n=418)$. Longitudinal multivariate analysis was used to evaluate blood pressure trajectories from pregnancy through 12 weeks postpartum.

Results-Obese and overweight women had significantly higher blood pressure readings as compared with women with normal BMI (all $p<0.05$ ). African American women who had high pregnancy weight gain had the greatest increase in mean arterial and diastolic blood pressures in pregnancy and postpartum.

Conclusion-Blood pressure trajectories in pregnancy and postpartum are significantly affected by race, BMI, and weight gain. Given the young age of this cohort, targeted efforts must be made for postpartum weight reduction to reduce cardiovascular risk.

\section{Keywords}

pregnancy; weight gain; obesity; racial differences

\begin{abstract}
African American women have higher rates of hypertension, cardiovascular disease (CVD) and mortality compared with other women living in the United States across all age groups. ${ }^{1,2}$ The ethnic disparity in hypertension is present in females from a young age with the black-to-white odds ratio of hypertension increasing from 2.11 to 4.04 between the ages of 15 and $65 .{ }^{3}$ The increased risk for CVD among African Americans has been associated with myriad factors such as higher body mass index (BMI), lower socioeconomic status, family history, poor dietary intake, physical inactivity, smoking, excess alcohol intake, and a history of hypertension in pregnancy. ${ }^{4}$ Hypertension in pregnancy and preeclampsia are present at even higher rates in African Americans than the baseline increase in hypertension outside of pregnancy. ${ }^{5,6}$ The important interaction between race and gestation-related
\end{abstract}

Copyright (C) 2012 by Thieme Medical Publishers, Inc.

Address for correspondence and reprint requests: Urania Magriples, M.D., Division of Maternal Fetal Medicine, Yale University School of Medicine, 333 Cedar Street, P.O. Box 208063, New Haven, CT 06520-8063 (urania.magriples@ yale.edu); reprints are not available from the author. 
hypertension and preeclampsia has been elucidated by previous research, but little is known about how race influences blood pressure changes in normotensive pregnant women. ${ }^{7,8}$

The physiological responsiveness of the cardiovascular system during pregnancy seems to impact the rate of cardiovascular events during pregnancy and the compliance of the cardiovascular system after pregnancy. The absence of normal vascular adjustment to pregnancy has been associated with complications such as hypertension and preeclampsia in late pregnancy. ${ }^{9-11}$ Blood pressure in healthy pregnant women decreases through weeks 20 to 26 of gestation, then steadily increases by 7 to $10 \%$ of the nadir until delivery, with blood pressure returning to baseline postpartum. Women who do not exhibit this physiological nadir but rather maintain a stable blood pressure in the first half of pregnancy and steadily increase until delivery have higher rates of pregnancy-induced hypertension and preeclampsia and higher rates of hypertension and ischemic heart disease later in life. ${ }^{12,13}$

In addition to higher initial blood pressure, African Americans have a higher rate of overweight and obesity, which may put additional strain on their cardiovascular system. According to the U.S. Centers for Disease Control and Prevention, $79.1 \%$ of non-Hispanic black women can be categorized as overweight or obese as compared with $59.4 \%$ of nonHispanic white women. ${ }^{14}$ Obesity in pregnancy has been associated with higher blood pressure and vascular dysregulation. ${ }^{15}$

The purpose of this study is to examine blood pressure trends for young pregnant women as well as the interaction of race and BMI. Understanding the factors that affect the differences in the physiological change in blood pressure during pregnancy could provide insight into the disparities seen in CVD and provide an opportunity for screening and early intervention with young women at risk for CVD.

\section{Materials and Methods}

\section{Study Population}

Data for this analysis are from a multisite randomized controlled trial following young pregnant women (ages 14 to 25) from early pregnancy to 1-year postpartum. Detailed methodology is described elsewhere. ${ }^{16}$ The prospective trial assessed the impact of individual versus group prenatal care on maternal behavior and birth outcomes. Women entering prenatal care between September 2001 and December 2004 were recruited from university-affiliated and county hospital public prenatal clinics in New Haven, Connecticut and Atlanta, Georgia. Inclusion criteria were: (1) pregnant less than 24 weeks gestation, (2) age 25 years or younger, (3) no severe medical problem requiring individualized assessment or tracking as a "high-risk pregnancy" (e.g., HIV), (4) ability to attend groups conducted in English or Spanish, and (5) willingness to be randomized to group versus individual prenatal care. Of 1,542 eligible young women, 1,047 enrolled in the study (68\% participation) of which 503 were in the New Haven cohort and 544 in the Atlanta cohort.

With a primary aim of assessing the impact of race on blood pressure changes in pregnancy, this secondary analysis includes only the more ethnically diverse New Haven cohort, as all of the women from Atlanta were African American. To compare differences among "healthy" young women, several additional exclusions in the New Haven sample were made: women with prepregnancy personal history of hypertension $(n=28)$, heart disease $(n$ $=11)$, diabetes $(n=19)$, family history of hypertension $(n=9)$, and women experiencing pregnancy-induced hypertension ( $n=33$ ) or gestational diabetes $(n=5)$. A total of 78 women (56 with one condition and 22 with more than one condition) were removed from this analytic data set. Additionally, because multiple gestation substantially affects blood pressure, cardiac output, and the risk of preeclampsia, twin pregnancies were excluded $(n=$ 
6). One woman who did not have any blood pressure readings across her pregnancy was also excluded. The resulting data set included 418 young women ages 14 to $25(82.7 \%$ of the Yale-New Haven cohort).

\section{Data Collection}

Data were collected by two methods: audio-computer-assisted self-interviewing and medical record review. Interview data were collected upon study entry prior to randomization (mean $=18.1$ weeks' gestation, standard deviation $[\mathrm{SD}]=3.3$ ) and again in the third trimester of pregnancy (mean $=34.5$ weeks' gestation, $\mathrm{SD}=3.3$ ). Participants were interviewed about demographic, psychosocial, and behavioral characteristics and were paid $\$ 20$ for each interview.

Medical record reviews were conducted by trained research staff. These reviews included abstraction of information from each prenatal care visit as well as events during labor and delivery. Overall, there were no differences between those reviewed and those lost to follow-up. ${ }^{16}$

\section{Measures}

Blood Pressure-Blood pressure readings for each participant throughout pregnancy were abstracted from medical records. All blood pressures where obtained electronically. Blood pressures above 140/80 were manually verified by a nurse in all cases. The primary outcome variables were repeated systolic blood pressure (SBP) and diastolic blood pressure (DBP) as well as mean arterial pressure (MAP) derived from the blood pressure readings: $\mathrm{MAP}=\mathrm{DBP}+1 / 3(\mathrm{SBP}-\mathrm{DBP})$. Each woman included in these analyses had at least 2 and up to 21 separate blood pressure readings recorded over the course of their pregnancy and 12 weeks postpartum $($ mean $=12.3$ blood pressure readings, $\mathrm{SD}=3.2$ ).

BMI and Weight Gain-Prepregnancy BMI was calculated from weight and height variables obtained from medical record data: weight $(\mathrm{kg}) / \mathrm{height}\left(\mathrm{m}^{2}\right)$. Based on the 2009 Institute of Medicine (IOM) Guidelines, participants were placed in one of four BMI categories: underweight/lean (BMI < 18.5), normal weight (BMI 18.5 to 24.9), overweight (BMI 25 to 29.9), and obese (BMI > 30). A continuous variable for weight change was created for each time point, calculated by subtracting a woman's prepregnancy weight from her current weight. Thus, weight change was modeled as a time-varying predictor with high weight gain modeled in the figures as $1 \mathrm{SD}$ above the sample's average estimated weight change.

Race-The study focused on differences in blood pressure trajectories of African American women $(n=244)$ during pregnancy compared with their non-African American $(n=174)$ counterparts. Of those categorized as non-African American, 112 were Latina, 55 were white, and seven were of another race, reflecting the demographic distribution in the prenatal care setting. Although the non-African American group included women of multiple racial/ethnic backgrounds, we choose to include these women in a single group because (1) it was not possible to make multiple group comparisons due to the small numbers of white and "other" ethnicity women and (2) given that all of the women in this study were recruited from a publicly funded clinic, the non-African American women were similar with respect to the psychosocial and socioeconomic factors that may be relevant to blood pressure.

Stress-Because blood pressure is directly affected by stress, four types of stress were evaluated. The workload indicator addressed possible effects of work and school as a stressor. This variable contained five categories: working and in school, working full-time 
and not in school, working part-time and not in school, in school only, and neither working nor in school. Social conflict was based on the seven items of the social conflict subscale of the Social Relationship Scale. ${ }^{17}$ This subscale assessed the perceived degree of social conflict in an individual's everyday social network. The 10-item Perceived Stress Scale was used as an indicator of subjective stress. ${ }^{18}$ This scale assessed the degree to which individuals perceived situations in their lives to be stressful. Finally, prenatal distress was measured using the Revised Pregnancy Distress Questionnaire and included 17 items asking participants to rate how "bothered, worried, or upset" they were about various aspects of pregnancy. ${ }^{19}$ All psychosocial outcomes were measured during the third trimester of pregnancy.

Behavioral and Demographic Indicators-Substance use (cigarette, marijuana, illicit drugs, and alcohol use) was characterized at two time frames: (1) during pregnancy and (2) during year prior to pregnancy. Nutrition and exercise during pregnancy were also tested as potential predictors. A modified version of the Pregnancy-Relevant Health Behaviors Scale, which included all items on the exercise and nutrition subscales, was used to evaluate the scope of health-promoting behaviors pursued. ${ }^{19}$ Finally, demographic variables included maternal age, socioeconomic status (as defined by median family income), education completion (still in school/high school diploma versus dropout), and number of prior pregnancies (never before pregnant versus pregnant previously).

Statistical Methods-Multilevel modeling was used for the blood pressure trajectory analyses outlined herein. ${ }^{20}$ This approach is advantageous because it accounts for the nonindependence of the repeated measures and permits the modeling of average or "typical" blood pressure trajectories for each subgroup of interest. Separate mean trajectories predicting MAP, SBP, and DBP across pregnancy were calculated. Gestational age was a repeated measure (i.e., level 1 predictor), serving as the time variable accounting for whether and how blood pressure changes across pregnancy. Weight change was a level 1 timevarying predictor. Person-level (i.e., level 2) predictors such as race and prenatal distress were also examined and permitted an examination of how trajectories significantly change when individual difference variables are accounted for in the models. Models were built by first testing and defining the linear, quadratic, and cubic effects of time as a function of gestational age and as assessed by their Wald statistics and improvement to the Bayesian information criterion. Using these criteria, the MAP, DBP, and SBP trajectory models were found to be best represented in the final models as quadratic trajectories with the linear effect of time treated as a random effect. All other predictors were first individually tested and any significant main effects or interactions were then tested in a combined model. Effects were only retained in the final model if they maintained a significant Wald statistic $(p<0.05)$ and also significantly improved the Bayesian information criterion statistic.

\section{Results}

Demographic characteristics of the sample are presented in Table 1. The cohort represented a young, economically and socially disadvantaged group with normal blood pressure at initiation of care. Age ranged from 14 to 25, with a mean age of 20 years. On average, participants had $\sim 11$ years of education. Over $60 \%$ of participants exhibited excessive weight gain during pregnancy by IOM guidelines.

In the primary predictor analysis and in the final model, blood pressure was a function of gestational age. The results showed a significant linear $(p<0.0001)$ and quadratic function $(p<0.0001)$ of gestational age for MAP, SBP, and DBP, indicating that blood pressure had an initial dip and then slowly increased across the second half of pregnancy, which is consistent with prior research. The cubic function was initially a significant effect in the 
MAP and SBP models but in both cases became nonsignificant after the significant interaction terms were added to the models. Hence, only the linear and quadratic functions of time were included in the final models.

In the primary predictor analysis, African American women exhibited statistically significant higher blood pressure readings throughout pregnancy as compared with the other ethnic groups. This main effect of race was qualified by important interactions with linear time and weight change in the MAP model and weight change in the SBP and DBP models (Tables 2, 3, and 4). In the final models, no significant interactions were found with economic or behavioral variables. Prenatal distress had a significant effect on the SBP model but not on MAP or DBP. Race, BMI group, and weight change were the only consistently significant factors influencing blood pressure across the models.

There were statistically significant differences in blood pressure readings throughout pregnancy across BMI categories. Compared with normal-weight women, obese and overweight women had significantly higher blood pressure readings and underweight/lean women had significantly lower blood pressure readings on all indicators: MAP, SBP, and DBP (all $p<0.05$ ). Based on the estimated trajectory, blood pressure nadirs occurred at approximately midpregnancy, although the specific estimates varied as a function of the individual blood pressure measure as well as a function of what predictors were retained in the final models.

Figure 1 illustrates changes in MAP, SBP and DBP stratified by racial group and weight change pattern. With regard to mean arterial pressure (Fig. 1, top panel), there was blunting of the blood pressure nadir for African American women (significant race by linear time interaction, $\mathrm{b}=0.10, p<0.05$ ). The MAP trajectories were also qualified by significant weight change by race $(\mathrm{b}=-0.10, p<0.0001)$ and weight change by linear time $(\mathrm{b}=0.002$, $p<0.05)$ interactions. As clearly seen in the MAP figure, African American women who had high weight gain across their pregnancies had the highest initial MAP and the greatest increase in MAP across and beyond pregnancy.

With regard to SBP, there was not a significant interaction of race with either time or weight gain; there was only a significant interaction of weight gain by linear time and weight gain by quadratic time such that women who had high weight gain across pregnancy had significantly higher SBP across pregnancy, especially prior to childbirth.

Finally with regard to DBP, there was a significant race by weight change interaction $(\mathrm{b}=$ $-0.05, p<0.05)$ as well as a significant weight change by time interaction $(b=-0.002, p<$ $0.05)$. As in the MAP models, African American women who had high weight gain consistently had the highest blood pressure readings across pregnancy and postpartum. African American women with normal weight gain had a DBP trajectory similar to that of non-African American women, although their nadir was comparatively blunted. Blood pressure did not return to baseline in the time frame studied, 12 weeks post-partum. The highest blood pressure effect was seen in African American women with high antepartum weight gain.

\section{Discussion}

Hypertension and abnormal blood pressure variation in the second trimester can lead to pregnancy complications and are associated with chronic disease risk. Understanding the nature of blood pressure change in pregnancy may provide important information as we seek to understand determinants of the high rate of CVD in young African American women. 
Our results suggest that race, BMI, and excessive weight gain affect blood pressure and the pattern of blood pressure change throughout the course of pregnancy among young women. In effect, both the between-person (BMI group) and within-person (weight change) weight variables were uniquely predictive of higher blood pressure readings across pregnancy. Moreover, high weight gain was also associated with a blunted nadir. In addition, African American women had poorer blood pressure trajectories across the models.

This finding of a difference in blood pressure trajectory by BMI supports prior research suggesting that women with BMI $>30$ display diminished vascular responsiveness during pregnancy. ${ }^{21}$ Women with excessive weight gain in pregnancy have higher baseline blood pressure, with a blunting of the physiological "dip" of pregnancy, and reset their blood pressure at a higher level in the postpartum period. Prior studies have demonstrated that pregnant women who develop preeclampsia have less of a "dip" and elevated blood pressure throughout pregnancy. Our study shows that in normotensive pregnancies not complicated by preeclampsia or gestational hypertension, the physiological dip of pregnancy and the overall trajectory of blood pressure is absent in African Americans and patients with excessive weight gain. Blood pressure did not return to baseline in the postpartum period as expected-an effect most prominent in African Americans and patients with excessive weight gain.

Our study has a few limitations, some of which may underestimate the results. Categorizations for maternal weight are based on categories for adult women (> 18 years), therefore our "underweight/lean" may actually be "normal" for young teens and likewise, our teens categorized as "normal" may be "overweight" in reality. This limitation may attenuate our results. Postnatal factors such as postpartum depression, parenting stress, sleep deprivation, and/or nutritional changes were not assessed and could have a significant impact on blood pressure. Prenatal distress may not directly correlate with postnatal distress and further study is needed to delineate which social, behavioral, and nutritional factors impact the rise in postpartum blood pressure. Though overall blood pressure readings were within the normal range, blood pressure changes observed are statistically and clinically significant. These results must be considered in the context of young women who are in the beginning of their reproductive lives. Whether or not future pregnancies and further weight gain represent a cumulative risk must be further investigated.

These analyses support prior evidence of higher blood pressure for African American women compared with non-African American women and extends these results to young low-risk pregnant women. Additionally, we address the association between BMI and weight gain during pregnancy on blood pressure and on the pattern of blood pressure change during pregnancy. Results suggest that the physiological nadir may be blunted for overweight and obese women. The mechanism by which obesity affects blood pressure in pregnancy is not clearly understood. Proposed mechanisms include dysregulation of the autonomic nervous system, adiposity-related insulin resistance, increased oxidative stress, chronic inflammation, and decreased endothelial responsiveness. ${ }^{22,23}$ Reduced nitric oxide bioavailability has been recognized as a hallmark of preeclampsia and low nitric oxide bioavailability has been reported in nonpregnant black women. ${ }^{24}$ Normotensive pregnant black women have also been shown to have reduced nitric oxide synthesis and altered vascular reactivity. ${ }^{25}$ This blunted vascular response in African American women may account for baseline differences in blood pressure that are negatively impacted by obesity.

Of significant concern in this young population is that blood pressure continues to rise during the study period up to 12 weeks postpartum and that this rise also correlates with race and weight gain. If each pregnancy resets baseline weight and blood pressure in this young cohort of women, their future reproductive and cardiovascular risks are likely to increase. 
Longitudinal studies are needed to elucidate the cumulative risks of pregnancy and obesity on the cardiovascular system as well as interventions targeting women in the interconceptual period to counteract these risks.

\section{Acknowledgments}

This research was funded by NIMH R01 MH/HD61175 to Jeannette R. Ickovics, Ph.D.

\section{References}

1. Jones-Webb R, Yu X, O'Brien J, Hannan P, Wall M, Oswald J. Does socioeconomic position moderate the effects of race on cardiovascular disease mortality? Ethn Dis. 2004; 14:489-496. [PubMed: 15724767]

2. Cutler JA, Sorlie PD, Wolz M, Thom T, Fields LE, Roccella EJ. Trends in hypertension prevalence, awareness, treatment, and control rates in United States adults between 1988-1994 and 1999-2004. Hypertension. 2008; 52:818-827. [PubMed: 18852389]

3. Geronimus AT, Bound J, Keene D, Hicken M. Black-white differences in age trajectories of hypertension prevalence among adult women and men, 1999-2002. Ethn Dis. 2007; 17:40-48. [PubMed: 17274208]

4. Gillum RF. Epidemiology of hypertension in African American women. Am Heart J. 1996; 131:385-395. [PubMed: 8579038]

5. Dekkers JC, Snieder H, Van Den Oord EJ, Treiber FA. Moderators of blood pressure development from childhood to adulthood: a 10-year longitudinal study. J Pediatr. 2002; 141:770-779. [PubMed: 12461492]

6. Winkleby MA, Robinson TN, Sundquist J, Kraemer HC. Ethnic variation in cardiovascular disease risk factors among children and young adults: findings from the Third National Health and Nutrition Examination Survey, 1988-1994. JAMA. 1999; 281:1006-1013. [PubMed: 10086435]

7. Wilson BJ, Watson MS, Prescott GJ, et al. Hypertensive diseases of pregnancy and risk of hypertension and stroke in later life: results from cohort study. BMJ. 2003; 326:845-849. [PubMed: 12702615]

8. Funai EF, Friedlander Y, Paltiel O, et al. Long-term mortality after preeclampsia. Epidemiology. 2005; 16:206-215. [PubMed: 15703535]

9. Macdonald-Wallis C, Tilling K, Fraser A, Nelson SM, Lawlor DA. Established preeclampsia risk factors are related to patterns of blood pressure change in normal term pregnancy: findings from the Avon Longitudinal Study of Parents and Children. J Hypertens. 2011; 29:1703-1711. [PubMed: 21841545]

10. Hermida RC, Ayala DE, Iglesias M. Predictable blood pressure variability in healthy and complicated pregnancies. Hypertension. 2001; 38(3 Pt 2):736-741. [PubMed: 11566967]

11. Hermida RC, Ayala DE. Prognostic value of ambulatory blood pressure measurements for the diagnosis of hypertension in pregnancy. Expert Rev Cardiovasc Ther. 2004; 2:375-391. [PubMed: 15151484]

12. Gaillard R, Bakker R, Willemsen SP, Hofman A, Steegers EA, Jaddoe VW. Blood pressure tracking during pregnancy and the risk of gestational hypertensive disorders: the Generation $\mathrm{R}$ Study. Eur Heart J. 2011; 32:3088-3097. [PubMed: 21821845]

13. Lykke JA, Langhoff-Roos J, Sibai BM, Funai EF, Triche EW, Paidas MJ. Hypertensive pregnancy disorders and subsequent cardiovascular morbidity and type 2 diabetes mellitus in the mother. Hypertension. 2009; 53:944-951. [PubMed: 19433776]

14. CDC. [Accessed Jan 27, 2012] Health United States, 2010. Table 71. 2011. Available at: http:// www.cdc.gov/nchs/data/hus/hus10.pdf

15. Gaillard R, Steegers EA, Hofman A, Jaddoe VW. Associations of maternal obesity with blood pressure and the risks of gestational hypertensive disorders. The Generation R Study J Hypertens. 2011; 29:937-944. 
16. Ickovics JR, Kershaw TS, Westdahl C, et al. Group prenatal care and perinatal outcomes: a randomized controlled trial. Obstet Gynecol. 2007; 110(2 Pt 1):330-339. Erratum in: Obstet Gynecol 2007, 110, 937. [PubMed: 17666608]

17. O'Brien K, Wortman CB, Kessler RC, Joseph JG. Social relationships of men at risk for AIDS. Soc Sci Med. 1993; 36:1161-1167. [PubMed: 8511645]

18. Cohen, S.; Williamson, GM. Perceived stress in a probability sample of the United States. In: Spacapan, S.; Oskamp, S., editors. The Social Psychology of Health. Newbury Park, CA: Sage; 1988.

19. Lobel, M. The Revised Pregnancy Distress Questionnaire (NUPDQ). Stony Brook, NY: State University of New York at Stony Brook; 1996.

20. Singer, JD.; Willett, JB. Applied Longitudinal Data Analysis: Modeling Change and Event Occurrence. New York, NY: Oxford University Press; 2003.

21. Helmreich RJ, Hundley V, Varvel P. The effect of obesity on heart rate (heart period) and physiologic parameters during pregnancy. Biol Res Nurs. 2008; 10:63-78. [PubMed: 18647761]

22. Lee LV, Foody JM. Women and heart disease. Cardiol Clin. 2011; 29:35-45. [PubMed: 21257099]

23. Patrick TE, Hubel CA, Roberts JM. Evidence of increased oxidative stress, unexplained by lipid changes, is present in nulliparous black women from early gestation. Hypertens Pregnancy. 2004; 23:91-100. [PubMed: 15117603]

24. Mata-Greenwood E, Chen DB. Racial differences in nitric oxide-dependent vasorelaxation. Reprod Sci. 2008; 15:9-25. [PubMed: 18212350]

25. Brewster LM, Taherzadeh Z, Volger S, et al. Ethnic differences in resistance artery contractility of normotensive pregnant women. Am J Physiol Heart Circ Physiol. 2010; 299:H431-H436.

[PubMed: 20511407] 

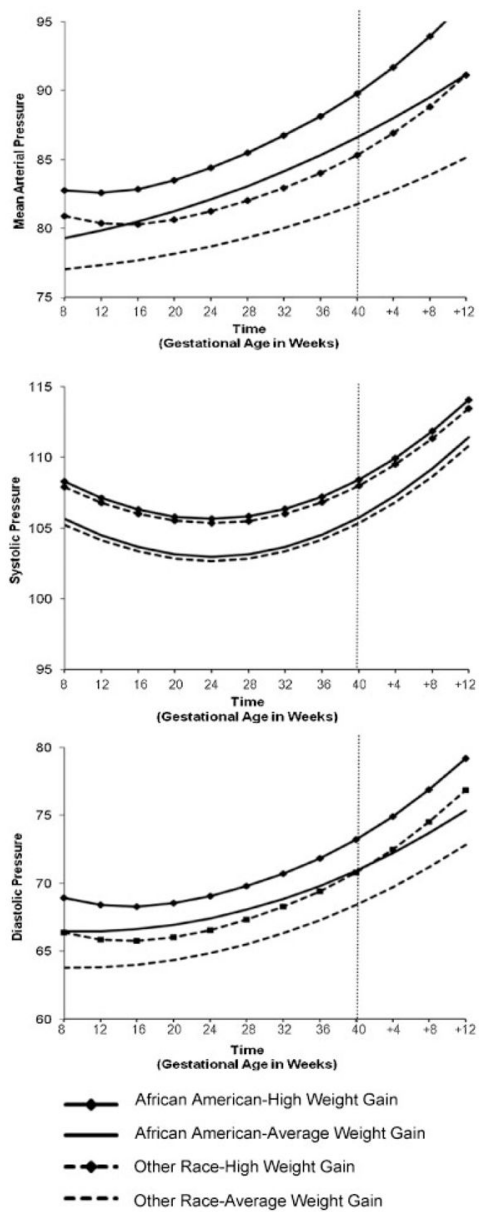

Fig. 1.

Estimated blood pressure trajectories by race and weight change. 


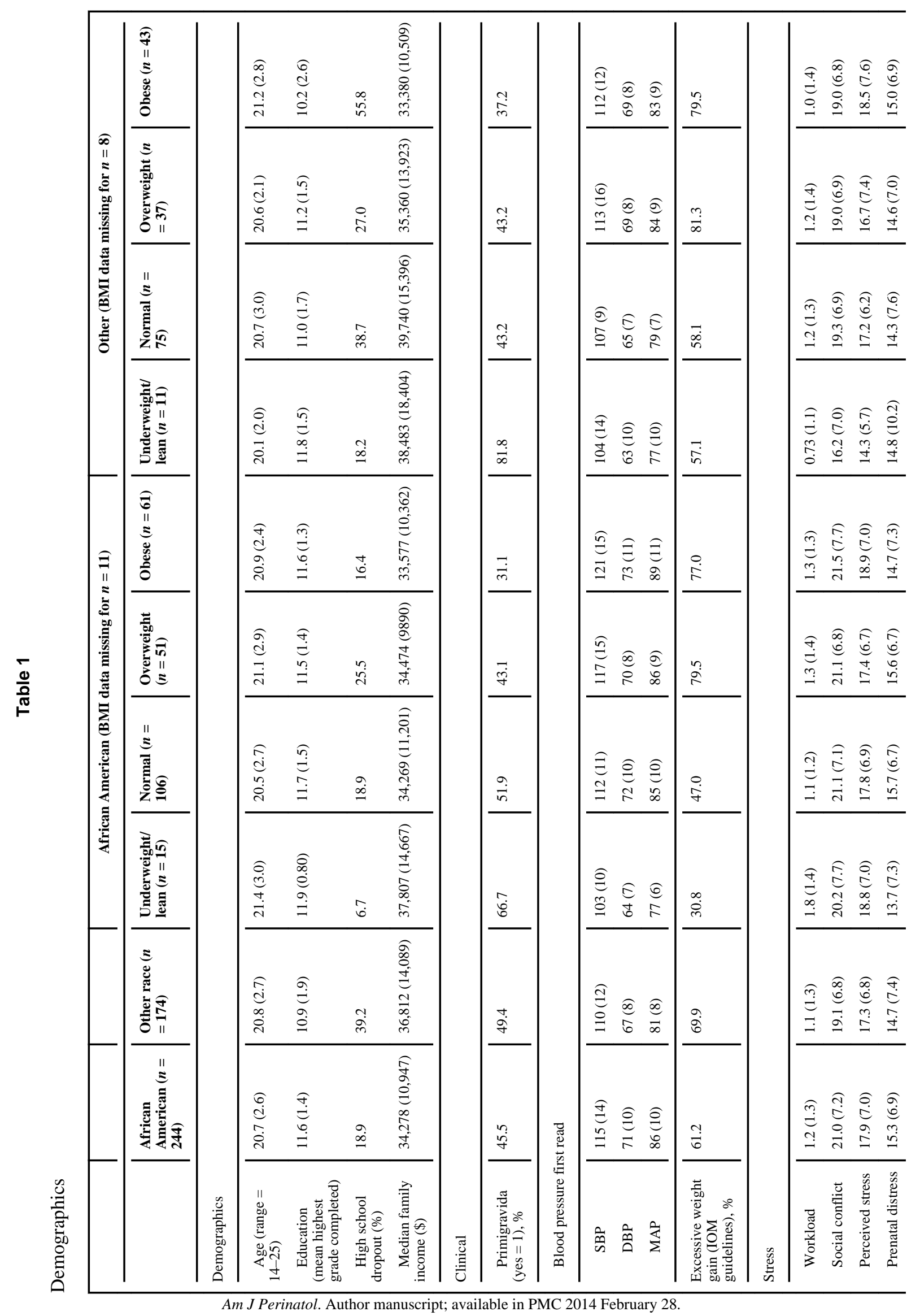




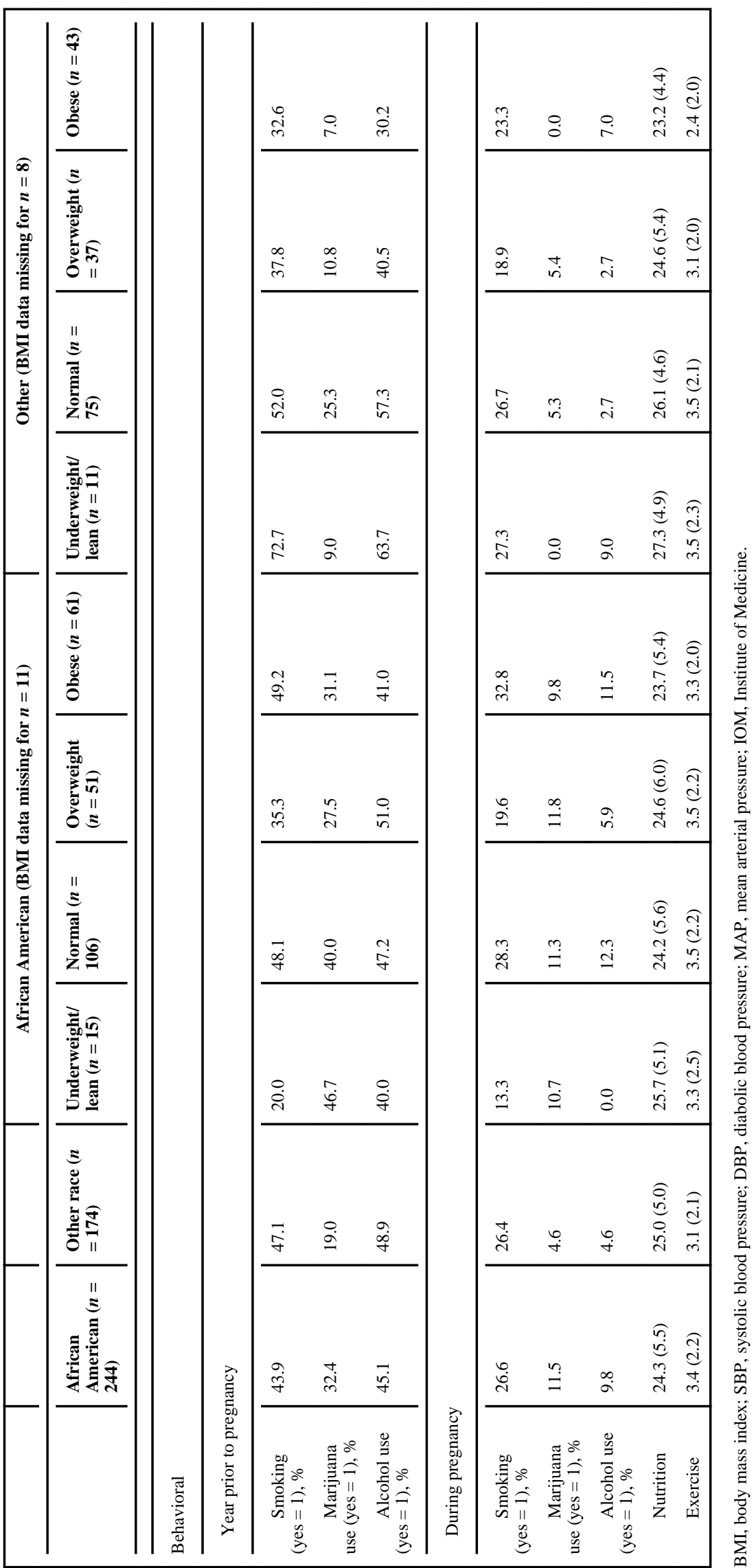




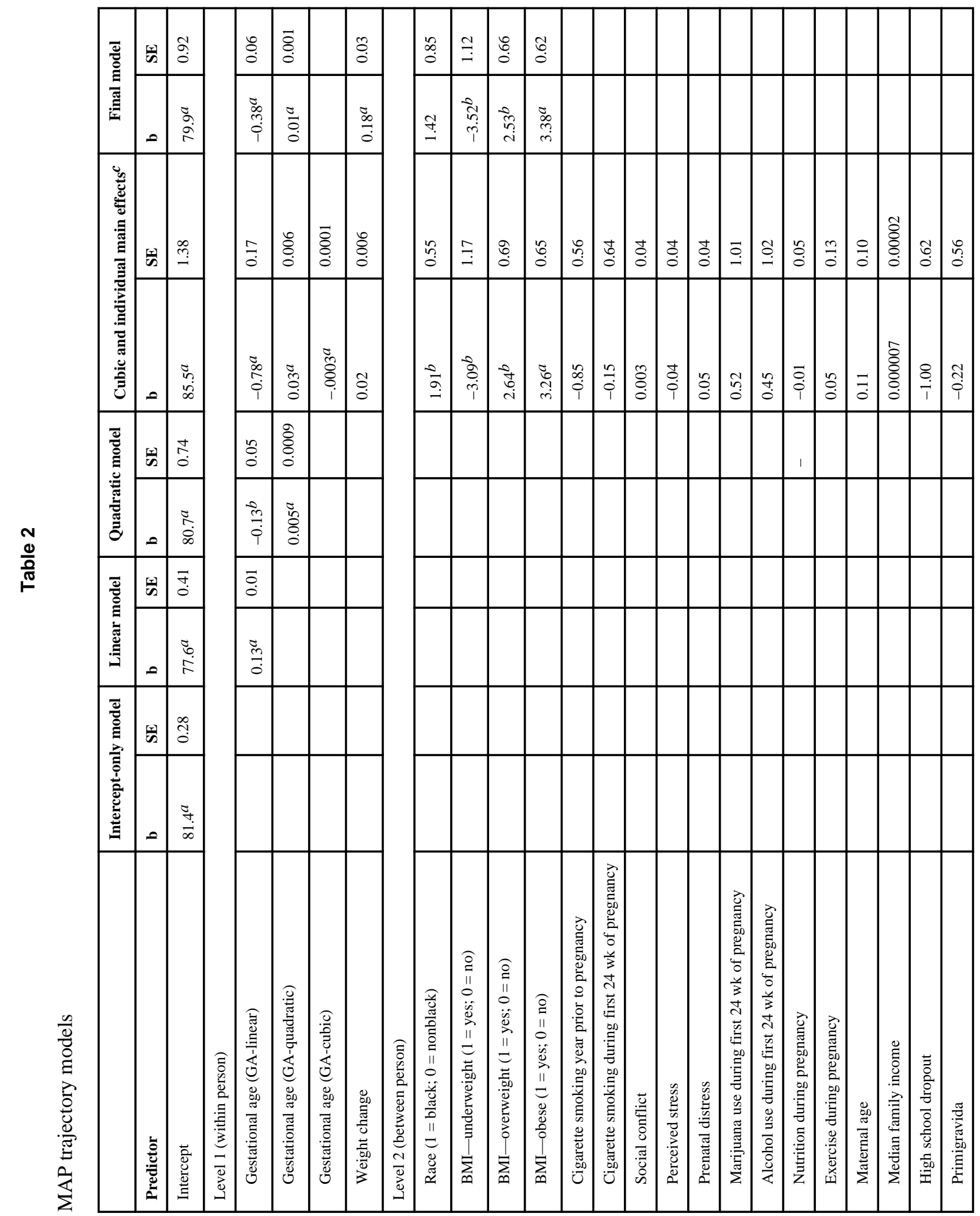




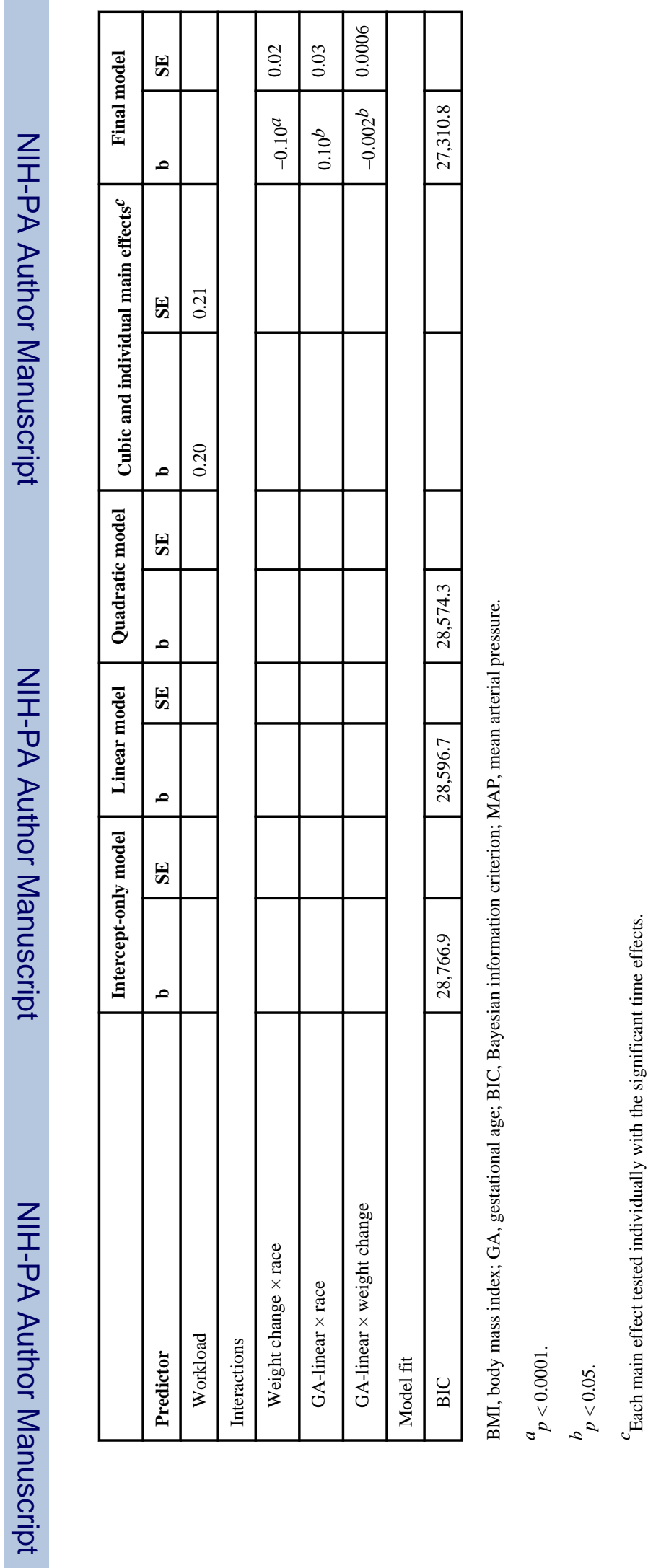

Am J Perinatol. Author manuscript; available in PMC 2014 February 28. 


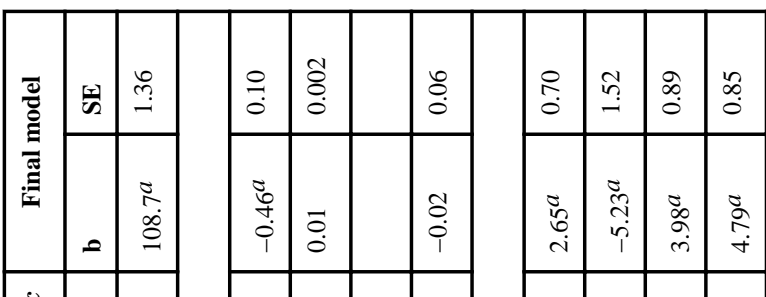

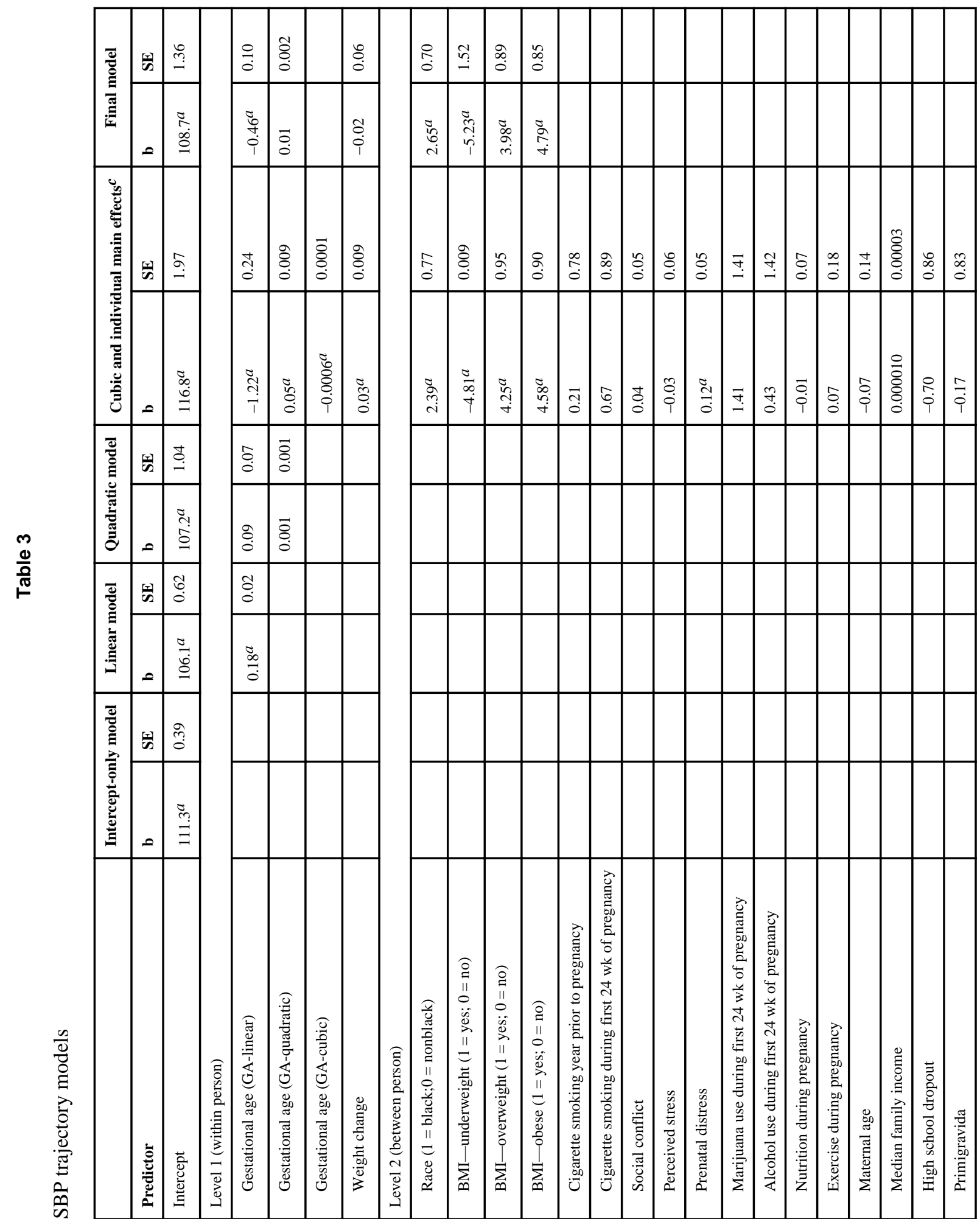




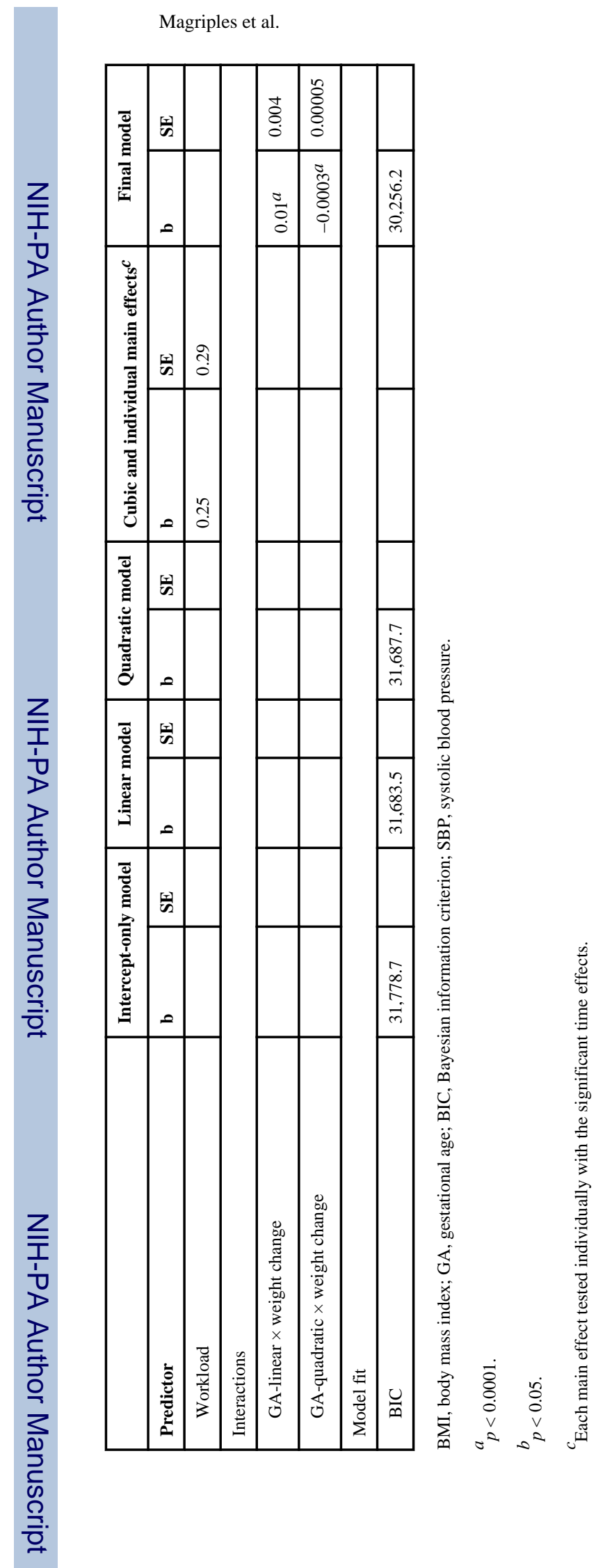

Am J Perinatol. Author manuscript; available in PMC 2014 February 28. 


\begin{tabular}{|c|c|c|c|c|c|c|c|c|c|c|c|c|c|c|c|c|c|c|c|c|c|c|c|}
\hline \multirow{2}{*}{ 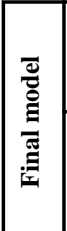 } & 䶕 & $\bar{\sigma}$ & $\stackrel{8}{0}$ & $\overrightarrow{8}$ & & $\stackrel{\overbrace{}}{\circ}$ & $\stackrel{\widetilde{\sigma}}{\circ}$ & $\stackrel{\grave{o}}{-}$ & $\stackrel{8}{0}$ & के & I্ণ & & & & & & & & & & & & \\
\hline & $=$ & : & $\begin{array}{l}0 \\
0 \\
0 \\
i \\
i\end{array}$ & $\begin{array}{l}0 \\
\stackrel{0}{0} \\
\stackrel{0}{0}\end{array}$ & & 帘 & $\begin{array}{l}\infty \\
\stackrel{\infty}{i} \\
i\end{array}$ & $\begin{array}{l}\infty \\
0 \\
i \\
i\end{array}$ & in & $\begin{array}{l}0 \\
\bar{n} \\
\vec{i}\end{array}$ & $\stackrel{\widetilde{F}}{\rightarrow}$ & & & & & & & & & & & & \\
\hline 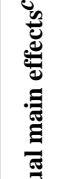 & 秉 & $\stackrel{\stackrel{n}{n}}{\rightarrow}$ & $\frac{\infty}{0}$ & $\begin{array}{l}\hat{\delta} \\
\dot{0} \\
\dot{0}\end{array}$ & $\begin{array}{l}\bar{\Xi} \\
\vdots \\
\circ\end{array}$ & ¿̊ & $\bar{n}$ & $\exists$ & $\stackrel{n}{0}$ & $\stackrel{\Xi}{\circ}$ & $\bar{n}$ & ڤે & o̊ & $\stackrel{t}{\circ}$ & $\begin{array}{l}t \\
\stackrel{0}{0}\end{array}$ & ğ & $\stackrel{n}{o}$ & $\begin{array}{l}0 \\
0 \\
0\end{array}$ & $\stackrel{7}{0}$ & $\stackrel{0}{0}$ & $\left|\begin{array}{l}\tilde{\delta} \\
0 \\
0 \\
0\end{array}\right|$ & $\sqrt{\tilde{n}}$ & กิ \\
\hline 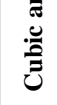 & $\therefore$ & $\begin{array}{l}\stackrel{\Xi}{\circ} \\
\stackrel{8}{8}\end{array}$ & $\begin{array}{c}\text { जे } \\
\text { wे } \\
\text { î }\end{array}$ & ठैّ & $\begin{array}{l}\overline{8} \\
0 \\
0 \\
1\end{array}$ & ¿̊. & $\stackrel{+}{+}$ & $\underset{i}{i}$ & $\stackrel{\infty}{\infty}$ & 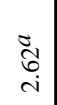 & $\underset{i}{\stackrel{F}{*}}$ & 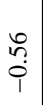 & $\begin{array}{l}\stackrel{\Omega}{0} \\
\stackrel{i}{0}\end{array}$ & $\begin{array}{l}n \\
\dot{i} \\
\dot{0}\end{array}$ & $\tilde{O}$ & $\stackrel{\infty}{0}$ & $\hat{3}$ & $\begin{array}{l}\overrightarrow{0} \\
\dot{i}\end{array}$ & 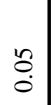 & $\begin{array}{l}\text { స్ } \\
\text { oे }\end{array}$ & $\mid \begin{array}{l}0 \\
0 \\
0 \\
0 \\
0\end{array}$ & $\stackrel{\text { in }}{i}$ & $\begin{array}{l}\text { त̃ } \\
\text { i }\end{array}$ \\
\hline
\end{tabular}

\begin{tabular}{|l|l|l|l|}
\hline \multirow{8}{*}{} & $\overrightarrow{0}$ & & \\
\hline & 0 & & \\
\hline
\end{tabular}

\begin{tabular}{llll|l|l|l|l|l|l|l|l|l|l|l|l|l|}
\hline & & & & & & & & & & & & & & & \\
\hline
\end{tabular}

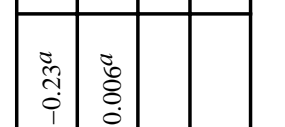

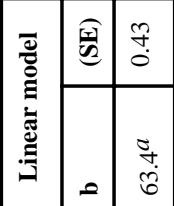
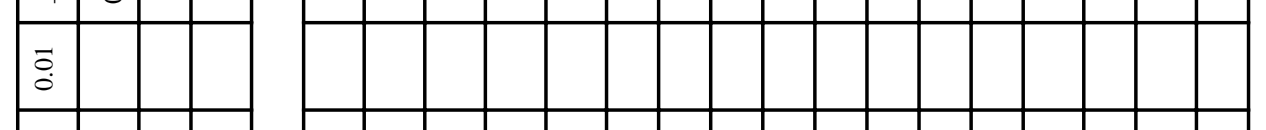

כ)

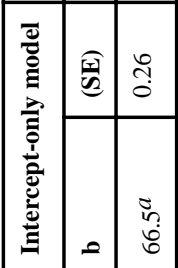
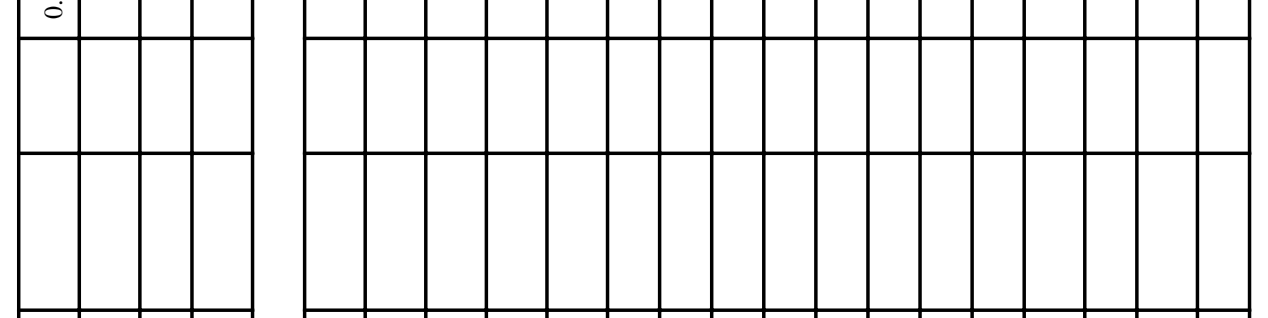


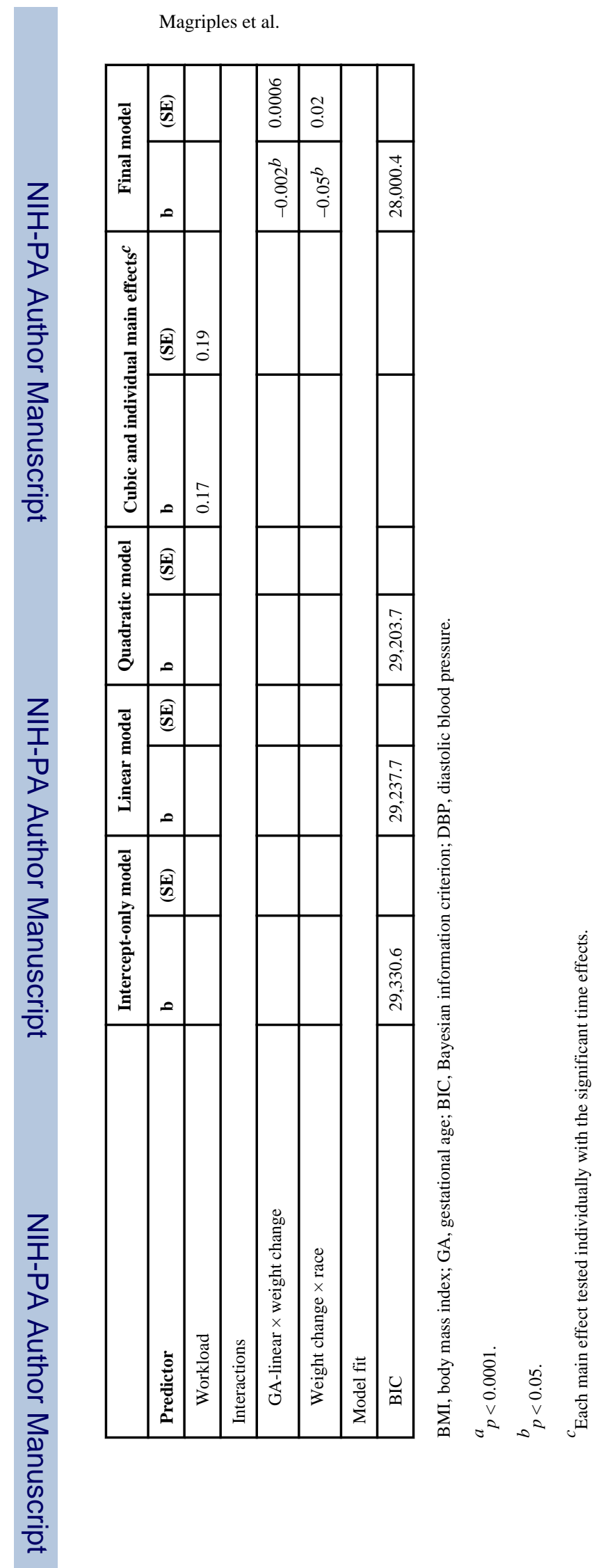

Am J Perinatol. Author manuscript; available in PMC 2014 February 28. 